\title{
Assessment of Nurses' Application of Guidelines for Management of Children Undergoing Hemodialysis
}

\author{
Walaa Hassan Ragab, Nursing Instructor \\ Pediatric Nursing, Technical Institute of Nursing, Alexandria University \\ Wafaa Mahmoud Abd-Elkader, Assistant Professor \\ Pediatric Nursing, Faculty of Nursing, Alexandria University \\ Hanan Mohamed Fathy, Assistant Professor \\ Pediatric Medicine, Faculty of Medicine, Alexandria University
}

Abeer Abd-El-Razik Ahmed, Assistant Professor
Pediatric Nursing, Faculty of Nursing, Alexandria University

Abstract

Chronic renal failure $(C R F)$ refers to progressive, irreversible impairment in renal function in which the body ability to maintain metabolic, fluid and electrolytes balance fails. Hemodialysis (HD) is the most common method used to treat CRF. Objective: Assess nurses' application of guidelines for management of children undergoing HD. Setting: This study was conducted at the Kidney Dialysis Unit at Smouha University Children's Hospital. Subjects: All nurses who are responsible for providing direct care for children undergoing HD admitted to the previously mentioned setting. Tool: One tool was used; Nurses' Application of Guidelines for Management of Children Undergoing Hemodialysis Observational Checklist. Results: Findings of the present study revealed that all nurses had "satisfactory" application scores about guidelines for management of children before, during and after HD as well as infection control precautions and documentation (100\% for each). Conclusion: All nurses had "Satisfactory" application score about guidelines for management of children undergoing HD. Recommendations: Booklet about recent guidelines for management of children undergoing HD procedure should be available in HD unit.

Keywords: Nurses' Application; Guidelines; Children; Hemodialysis.

\section{Introduction}

Chronic Renal Failure (CRF) is a devastating medical, social, and economic problem for children and their families (Brück et al., 2015). It progresses to End Stage Renal Disease (ESRD). At this stage, life can be sustained only with transplantation or dialysis (Kaspar et al., 2016). Worldwide, there are more than 30 in every 100,000 children are suffering from CRF each year in 2017 (Naritata et al., 2017).

The etiology of CRF in children may be due to congenital malformations as reflux nephropathy and polycystic kidney. Additionally, it can occur secondary to systemic disorders that impair kidney function as diabetes mellitus and hypertension (Ibrahim et al., 2019).

Chronic renal failure leads to severe complications as renal osteodystrophy, growth retardation, hypertension and cardiac dysfunction (pitman, 2013). It can be managed through transplantation or dialysis. Dialysis has two types; peritoneal dialysis and Hemodialysis (HD) (El Deeb, 2016).

Hemodialysis is the most common method used to treat CRF in children. It refers to a medical procedure that uses a special machine to filter waste products from the blood through a semi-permeable membrane (Kallenbach, 2015). In United States, the International Pediatric Nephrology Association (2018) estimated that 12.9 per million populations among 
children less than 19 years were treated by HD.

The success of HD depends on nursing care provided for children. The HD nurses play a pivotal role in providing information, care, support, and therapeutic counseling to the pediatric patients and their families throughout the entire illness (Peimani \& Pajouhi, 2010). Being aware of the recent nursing guidelines for management of children undergoing HD will allow nurses to make an appropriate clinical decision (James et al., 2014).

\section{Aim of the Study}

The aim of the study is to assess the nurses' application of guidelines for management of children undergoing HD.

\section{Research Question}

To what extent do nurses apply the guidelines for management of children undergoing $\mathrm{HD}$ ?

\section{Materials and Method}

\section{Materials}

Design: A descriptive research design was used to accomplish the study.

Setting: This study was conducted at the Kidney Dialysis Unit at Smouha University Children's Hospital.

Subjects: All nurses who were responsible for providing direct care for children undergoing $\mathrm{HD}$ admitted to the previously mentioned setting during a period of four months comprised the study subjects. Their number was 21 nurses.

\section{Tool:}

Tool I: Nurses' Application of Guidelines for Management of Children Undergoing Hemodialysis Observational Checklist

This tool was developed by the researcher guided by the Egyptian Society of Nephrology and Transplantation Hemodialysis Guidelines 2019, Centers for Disease Control and Prevention (CDC) in
2017 and relevant literature (Kimmel \& Rosenberg, 2020; Levy et al., 2016).

It was used to assess nurses' practices in the application of guidelines regarding to the management of children with CRF undergoing HD. It included the following:

Part I: Nurses' practices before the initiation of HD: as the preparation of environment, equipment and supplies, HD machine and children.

Part II: Nurses' practices during HD: as intradialytic patient assessment and machine monitoring, offering physical and psychological care.

Part III: Nurses' practices after the termination of HD: as termination of HD regarding environment, equipment and supplies, HD machine and children.

Part IV: Nurses' practices concerning infection control precautions: as washing hands, wearing personal protective equipment, discarding sharp items in appropriate container, disinfecting HD machine, dialyzing child with hepatitis B or $\mathrm{C}$ virus in a separate room with a separate machine.

Part V: Documentation before, during and after HD: Before HD as vital signs, investigations done, machine's data and body weight. During HD as vital signs, medication given. After $\mathrm{HD}$ as vital signs and body weight.

Nurses' characteristics, such as, age, level of education, years of experience, and attendance of training program about HD were attached to the tool.

\section{Method}

- Approval from the Ethical Research Committee of the Faculty of Nursing at Alexandria University was obtained.

- Official letter was directed to responsible authorities to take their permission to conduct the study after explaining its purpose. 
- The tool was developed and was tested for its content validity by five experts in Pediatric Nursing field.

- Reliability of the tool was asserted using Cronbach Coefficient Alpha Test, where $r=0.724$.

- A pilot study was conducted on 3 nurses to test the clarity and feasibility of the tool. Accordingly, the necessary modifications were done. Those nurses were excluded from the subject.

- Every nurse was asked individually about her characteristics in nurses' office during the break time.

- Every nurse was closely observed four times (two observations in the morning and two observations in the afternoon shifts) while performing HD (before, during and after HD).

- The mean of the four observations was taken.

- Data was collected over a period of four months starting from the beginning of July 2020 to the end of October 2020.

\section{Ethical considerations:}

- A formal witness written consent was obtained from the head nurse after explanation of the aim of the study.

- Confidentiality of participants' data was considered and privacy was ensured.

\section{Statistical Analysis}

Data were analyzed using the statistical package for social science SPSS (version 20). The level of significance selected for this study was $\leq 0.05$.

\section{Results}

Table (1) shows nurses application of guidelines according to their performance before HD. Regarding preparation of machine, all nurses primed lines and dialyzer with prescribed solution, set dialysate flow, heparin infusion time and rate, session length as well as blood pump (100\% of observations for each). Regarding preparation of the children, all nurses measured the children's body weight, blood pressure and assessed access site for signs of infection (100\% of observations for each). Regarding initiation of the HD, all nurses cleansed each catheter exits with $70 \%$ alcohol or $10 \%$ povidone iodine and withdraw previously injected heparin from them (100\% of observations for each).

Table (2) portrays nurses' application of guidelines according to their performance during HD. It all nurses measured blood pressure as well as observed and reported for occurrence of complications $(100 \%$ of observations for each). While, none of them checked clotting time in $100 \%$ of their observations.

Table (3) shows nurses' application of guidelines according to their performance after HD. Concerning children, all nurses flushed lumens of catheters with $10 \mathrm{ml}$ normal saline $0.9 \%$, instilled heparin into lumens of each catheter as well as measured blood pressure and body weight (100\% of observations for each). Regarding HD machine, all nurses cleansed the outer surface of machine with a bleach solution and performed chemical disinfection using citric acid after each session $(100 \%$ of observations for each).

Table (4) clarifies the nurses' application of guidelines according to infection control precautions before, during and after HD. All nurses washed their hands before and after performing HD in addition to after removal of gloves. All nurses wore sterile gloves before performing or terminating HD (100\% of observations for each). Conversely, none of them wore face shield before, during or after $\mathrm{HD}$ in $100 \%$ of their observations.

Figure (1) reveals that all nurses had "Satisfactory" application score about guidelines for management of children undergoing HD (100\%). 
Table (5) shows that there were no statistically significant differences between nurses' application total percent score of guidelines for management of children undergoing $\mathrm{HD}$ and their characteristics except concerning their years of experience.

\section{Discussion}

Chronic renal failure is a major health problem encountered among children worldwide. Hemodialysis is a widely used mode of RRT in infants and children with ESRD (Madiha et al., 2017). It is a highly stressful situation that requires specific skills to achieve optimal outcomes.

The present study revealed that all nurses prepared HD machine according to guidelines. This could be attributed to that the machine is considered the core of HD procedure and without its preparation, no HD could be delivered. This result is consistent with the result of Ahmed (2007) who found that all nurses attained good score in the preparation of HD machine.

It was found from the current study that all nurses in their observations measured blood pressure before, during and after HD. These findings might be due to the nurses' awareness about the occurrence of intradialytic complications as hypotension. These findings were in the same line with the findings of Ibrahim et al. (2019) who reported that the majority of the nurses had competent practice level regarding measuring blood pressure before, during and after HD.

The results of the present study revealed that all nurses measured body weight before and after HD. These findings may be explained that HD nurses appreciate the importance of measuring body weight before HD to determine the ultrafiltration rate. Also, they measured it after HD to evaluate the efficiency of HD session. These results stand in opposition with the results of Hassona (2011) who reported that all nurses had unsatisfactory practice score regarding measuring body weight during HD.
The findings of the present study showed that all nurses assessed the catheters for infection before initiating HD, cleansed catheters exit with $70 \%$ alcohol or $10 \%$ povidone iodine, injected heparin into catheters after terminating HD. These findings could be related to nurses' awareness that keeping access site patent and infection free ensures that HD will go smoothly without complications. Similar findings were reported by Abdalmajed (2017).

The result of the present study reflected that all nurses used citric acid in the disinfection of the HD machine after HD. This result can be attributed to that nurse usually follow the policy of their HD unit. Similar result was reported by Ebrahem (2009) who found that all nurses rinsed fluid pathway after every HD session with citrosteril.

Hand decontamination should be performed before the initiation of $\mathrm{HD}$ procedure, during the HD procedure, after removal of gloves and after touching children's surroundings (Eltantawy, 2021). The current study findings revealed that all nurses decontaminated their hands before the HD procedure, after terminating HD procedure as well as after removal of the gloves. This might be due to the availability of hand wash facilities in HD. The findings of the present study are not supported by the findings of Ebrahem (2009) who found that the majority of nurses did not decontaminate their hands before HD. In addition, (Yanai et al. (2006) found that the minority of nurses washed their hands after removal of the gloves and after terminating HD procedure.

Personal protective equipment such as gloves, aprons and face shield is an important aspect of infection prevention (Brown, 2019). The current study showed that none of the nurses wore plastic apron during the HD or face shield before, during or after HD. This might be related to lack of such equipment. In this respect, Talaat et al. (2006) stated that the lack of infection control facilities was a major hindering 
factor to the application of infection control precautions in HD unit.

The result documented that all nurses wore sterile gloves before both initiating and terminating HD. These findings could be related to nurses' awareness about its importance in prevention of infection. These findings are contradicted with the findings of Ebrahem (2009) who found that the minority of nurses wore sterile gloves before both initiating and terminating HD.

The finding of the current study revealed that all nurses had "Satisfactory" mean score about application of guidelines for management of children undergoing HD. The result of the current study could be attributed to continuous supervision from the head nurse of the HD unit. This finding was not congruent with Ibrahim et al. (2019) who reported that more than half of nurses had incompetent level of total practice score regarding care of children undergoing HD.

\section{Conclusion}

Based on the findings of the present study, it is concluded that all nurses had "Satisfactory" application score about guidelines for management of children undergoing HD procedure.

\section{Recommendations}

- The recent guidelines for management of children undergoing HD should be applied by pediatric nurses in HD unit.

- Continuous training programs on application of guidelines for management of children undergoing HD procedure in HD unit.

- Booklet about recent guidelines for management of children undergoing HD procedure should be available in HD unit. 
Table (1): Nurses' application of guidelines according to their performance before HD

\begin{tabular}{|c|c|c|c|c|}
\hline \multirow{3}{*}{ Items } & \multicolumn{4}{|c|}{ Total $(n=84)$} \\
\hline & \multicolumn{2}{|c|}{ Done } & \multicolumn{2}{|c|}{ Not done } \\
\hline & No & $\%$ & No & $\%$ \\
\hline \multicolumn{5}{|l|}{ I. Preparation of the HD machine } \\
\hline - Priming all lines and the dialyzer with the prescribed solution & 84 & 100.0 & 0 & 0.0 \\
\hline - Checking the dialyzer and circuit for air bubbles & 84 & 100.0 & 0 & 0.0 \\
\hline Setting the dialysate flow & 84 & 100.0 & 0 & 0.0 \\
\hline - Setting heparin time and infusion rate & 84 & 100.0 & 0 & 0.0 \\
\hline - $\quad$ Setting session length & 84 & 100.0 & 0 & 0.0 \\
\hline - Setting the blood pump at $(150-200) \mathrm{m} / \mathrm{ml}$ & 84 & 100.0 & 0 & 0.0 \\
\hline \multicolumn{5}{|l|}{ II. Preparation of the children } \\
\hline - Measuring body weight & 84 & 100.0 & 0 & 0.0 \\
\hline - Measuring temperature & 84 & 100.0 & 0 & 0.0 \\
\hline Measuring pulse & 84 & 100.0 & 0 & 0.0 \\
\hline - Measuring respiration & 0 & 0.0 & 84 & 100.0 \\
\hline - Measuring blood pressure & 84 & 100.0 & 0 & 0.0 \\
\hline - Assessing access site for signs of infection & 84 & 100.0 & 0 & 0.0 \\
\hline III. Initiation of the HD procedure & & & & \\
\hline - Cleansing each catheter exit with $70 \%$ alcohol or $10 \%$ povidone iodine & 84 & 100.0 & 0 & 0.0 \\
\hline - Unclamping catheters and withdraw injected heparin from them & 84 & 100.0 & 0 & 0.0 \\
\hline - $\quad$ Priming each catheter with $10 \mathrm{ml}$ normal saline $0,9 \%$. & 84 & 100.0 & 0 & 0.0 \\
\hline - Connecting the blood lines to each catheter & 84 & 100.0 & 0 & 0.0 \\
\hline
\end{tabular}

Table (2): Nurses' application of guidelines according to their performance during hemodialysis

\begin{tabular}{||l|c|c|c|c||}
\hline \multirow{2}{*}{ Ittems } & \multicolumn{4}{|c||}{ Total (n=84) } \\
\cline { 2 - 5 } & \multicolumn{3}{|c|}{ Done } & \multicolumn{2}{c||}{ Not done } \\
\cline { 2 - 5 } & No. & $\mathbf{\%}$ & No. & \% \\
\hline I. Intradialytic patient's assessment and HD machine monitoring & & & & \\
\hline$-\quad$ Measuring temperature hourly & 84 & 100.0 & 0 & 0.0 \\
\hline Measuring pulse hourly & 84 & 100.0 & 0 & 0.0 \\
\hline Measuring respiration hourly & 0 & 0.0 & 84 & 0.0 \\
\hline Measuring blood pressure hourly & 84 & 100.0 & 0 & 0.0 \\
\hline- Checking clotting time hourly & 0 & 0.0 & 84 & 0.0 \\
\hline Monitoring of vascular access and lines connection & 78 & 92.9 & 6 & 7.1 \\
\hline- Giving prescribed medication as heparin and iron & 84 & 100.0 & 0 & 0.0 \\
\hline- Observing for occurrence of complications as: muscle cramps & 84 & 100.0 & 0 & 0.0 \\
\hline
\end{tabular}


Table (3): Nurses' application of guidelines according to their performance after hemodialysis

\begin{tabular}{|c|c|c|c|c|}
\hline \multirow{3}{*}{ |Items } & \multicolumn{4}{|c|}{ Total $(n=84)$} \\
\hline & \multicolumn{2}{|c|}{ Done } & \multicolumn{2}{|c|}{ Not done } \\
\hline & No. & $\%$ & No. & $\%$ \\
\hline \multicolumn{5}{|l|}{ I. Termination of procedure as regards children } \\
\hline Connecting prescribed solution to the blood lines & 84 & 100.0 & 0 & 0.0 \\
\hline Turning on solution to return blood to children through arterial line & 84 & 100.0 & 0 & 0.0 \\
\hline Turning on blood pump to return blood to children through venous line & 84 & 100.0 & 0 & 0.0 \\
\hline Disinfecting the catheter moving from the hub toward the body & 84 & 100.0 & 0 & 0.0 \\
\hline Disconnecting the blood line from the catheter & 84 & 100.0 & 0 & 0.0 \\
\hline Flushing the lumens of the catheters with $10 \mathrm{ml}$ of normal saline $0.9 \%$ & 84 & 100.0 & 0 & 0.0 \\
\hline Instilling heparin into each catheter & 84 & 100.0 & 0 & 0.0 \\
\hline Measuring temperature & 84 & 100.0 & 0 & 0.0 \\
\hline Measuring pulse & 84 & 100.0 & 0 & 0.0 \\
\hline Measuring respiration & 0 & 0.0 & 84 & 100.0 \\
\hline Measuring blood pressure & 84 & 100.0 & 0 & 0.0 \\
\hline Measuring body weight & 84 & 100.0 & 0 & 0.0 \\
\hline \multicolumn{5}{|l|}{ II. Termination of procedure as regards machine } \\
\hline $\begin{array}{l}\text { Disconnecting the used blood tubing and dialyzer from dialysis } \\
\text { machine }\end{array}$ & 84 & 100.0 & 0 & 0.0 \\
\hline Cleansing outer surface of machine with a bleach solution & 84 & 100.0 & 0 & 0.0 \\
\hline Performing chemical disinfection using citric acid after each session & 84 & 100.0 & 0 & 0.0 \\
\hline
\end{tabular}

Table (4): Nurses' application of guidelines according to infection control precautions before, during and after hemodialysis procedure

\begin{tabular}{|c|c|c|c|c|}
\hline \multirow{3}{*}{ Items } & \multicolumn{4}{|c|}{ Total $(n=84)$} \\
\hline & \multicolumn{2}{|c|}{ Done } & \multicolumn{2}{|c|}{ Not done } \\
\hline & No. & $\%$ & No. & $\%$ \\
\hline \multicolumn{5}{|l|}{ Before the initiation of HD procedure } \\
\hline Washing hands & 84 & 100.0 & 0 & 0.0 \\
\hline Wearing sterile gloves & 84 & 100.0 & 0 & 0.0 \\
\hline Wearing plastic apron & 80 & 95.2 & 4 & 4.8 \\
\hline$-\quad$ Wearing face shield & 0 & 0.0 & 84 & 100.0 \\
\hline \multicolumn{5}{|l|}{ During the HD procedure } \\
\hline Washing hands before any contact with children & 3 & 3.6 & 81 & 96.4 \\
\hline Changing sterile gloves between children & 84 & 100.0 & 0 & 0.0 \\
\hline Wearing plastic apron before any contact with children & 0 & 0.0 & 84 & 100.0 \\
\hline Wearing face shield before any contact with children & 0 & 0.0 & 84 & 100.0 \\
\hline \multicolumn{5}{|l|}{ After the HD procedure } \\
\hline Washing hands after terminating procedure & 84 & 100.0 & 0 & 0.0 \\
\hline Washing hands after removal of gloves & 84 & 100.0 & 0 & 0.0 \\
\hline Wearing sterile gloves before terminating procedure & 84 & 100.0 & 0 & 0.0 \\
\hline Wearing plastic apron before terminating procedure & 80 & 95.2 & 4 & 4.8 \\
\hline Wearing face shield before terminating procedure & 0 & 0.0 & 84 & 100.0 \\
\hline
\end{tabular}




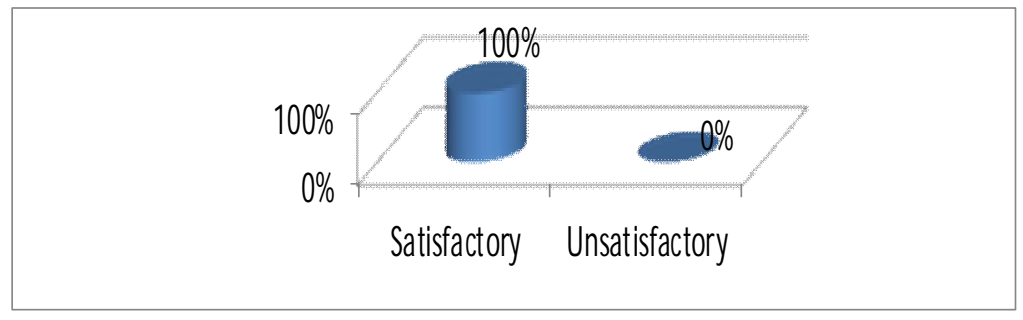

Figure (1): Total percent score of nurses' application of guidelines for management of children undergoing hemodialysis procedure

Table (5): Relation between nurses' application total percent score of guidelines for management of children undergoing hemodialysis procedure and their characteristics

\begin{tabular}{|c|c|c|}
\hline \multirow{2}{*}{ Items } & Total $(n=21)$ & \multirow[t]{2}{*}{ Test of Significance } \\
\hline & Mean \pm SD & \\
\hline \multicolumn{3}{|l|}{ Age (years) } \\
\hline$-<25$ & $132.00 \pm 0.000$ & \multirow{4}{*}{$\begin{array}{l}F=0.526 \\
P=0.670\end{array}$} \\
\hline$-25-$ & $130.84 \pm 1.952$ & \\
\hline$-35-$ & $131.21 \pm 1.503$ & \\
\hline$-\geq 45$ & $132.17 \pm 1.267$ & \\
\hline \multicolumn{3}{|l|}{ Level of education } \\
\hline - Secondary school of nursing diploma & $132.75 \pm 0.500$ & \multirow{3}{*}{$\begin{array}{l}F=0.601 \\
P=0.559\end{array}$} \\
\hline - Technical institute of nursing diploma & $130.96 \pm 1.513$ & \\
\hline - Bachelor degree of nursing & $131.46 \pm 2.206$ & \\
\hline \multicolumn{3}{|l|}{ Years of experience } \\
\hline$-<1$ & $131.56 \pm 1.780$ & \multirow{4}{*}{$\begin{array}{c}\mathrm{F}=3.356 \\
\mathrm{P}=0.044^{*}\end{array}$} \\
\hline$-1-$ & $128.13 \pm 1.959$ & \\
\hline$-5-$ & $131.13 \pm 0.991$ & \\
\hline$-\geq 10$ & $131.56 \pm 0.878$ & \\
\hline
\end{tabular}

F ANOVA test $\quad *$ Statistically significant at $p \leq 0.05$ 


\section{References}

- Abdalmajed, A.A.B. (2017). Nurses' Knowledge and Practice Regarding Nursing Care of Hemodialysis Catheter in Pediatric Renal Units in Khartoum. (Unpublished master thesis), Faculty of Nursing, National Ribat University.

- Ahmed, A. (2007). Assessment of Nursing Care provided for Children undergoing Hemodialysis. (Master degree), Faculty of Nursing, Alexandria University, unpublished thesis. PP. 87-92.

- Brown, L. (2019). Use of personal protective equipment in nursing practice. Nursing Standard, 34(5), 5966.

- $\quad$ Brück, K., Stel, V., \& Fraser, S. (2015). Translational research in nephrology: Chronic kidney disease prevention and public health. Clinical Kidney Journal, 8(1), 647-655.

- Centers for Disease Control and Prevention, (2017). Dialysis Safety. Retrieved from: https://www.cdc.gov/dialysis/preventio n-toSols/audit-tools.html.

- Ebrahem, G.G.S. (2009). Nurses' Application of Infection Control Precautions in Hemodialysis Unit at ElMansoura University Children's Hospital. (Master degree), Faculty of Nursing, Alexandria University, unpublished thesis.

- El Deeb, H.A.A. (2016). Effect of Reflexology Foot Massage on Fatigue Level and Leg Cramps for Patients on Hemodialysis. (Doctorate degree), Faculty of Nursing, Alexandria University, unpublished thesis.

- Eltantawy, H.F.E.A. (2021). Nurses' Knowledge versus their Application of Standard Infection Control Precautions in Pediatric Intensive Care Unit. (Master degree), Faculty of Nursing, Alexandria University, unpublished thesis.
- Gamal L, (2015). Establishing Standards for Prevention of Nosocominal Infection in The Recovery Rooms and Surgical Ward at ElMinia University Hospital. Unpublished Doctoral Thesis. Faculty of Nursing, Assiut University: 129.

- Hafez, M.H., Elsyed, H.M., Hassaballa, M., Fadel, F., \& Sarhan, I. (2020). The Egyptian Society of Nephrology and Transplantation Hemodialysis Guidelines 2019. Journal of Egyptian Society of Nephrology and Transplantation, 20(1). doi: 10.4103/jesnt.jesnt_44_19.

- Hassona, F.M.H. (2011). Evaluation of an Educational Program: A Report from the Hemodialysis Unit in Zagazig University Hospitals, Egypt. Nephrology Nursing Journal, 39- 53. Available from http://doi.org/10.9790/1959060506121 7.

- Ibrahim, M.A., Ouda, W.E., \& Ismail, S.S. (2019). Assessment of Nurses' Performance Regarding Care of Children Undergoing Hemodialysis Therapy. Egyptian Journal of Health Care, 10(3), 113-25.

- James, S. R., Nelson, K., \& Ashwill, J. (2014). Nursing Care of ChildrenEBook: Principles and Practice. Elsevier Health Sciences.

- Kallenbach, J. Z. (2015). Review of Hemodialysis for Nurses and Dialysis Personnel-E-Book. Elsevier Health Sciences.

- Kaspar, C., Bholah, R., \& Bunchman, T. (2016). Areview of pediatric chronic kidney disease. Blood Purification, (41): 211-17.

- Kimmel, P.L., \& Rosenberg, M.E. (2020). CHRONIC RENAL DISEASE. (2nd ed). United Kingdom: Elsevier Inc.

- Levy, J., Brown, E., \& Lawrence, A. (2016). Oxford Handbook of Dialysis. 
(4th ed). United Kingdom: Oxford University.

- Bayoumi, M., Mahmoud, N., (2017). Effect of education program on nurses' knowledge and practice regarding care of central venous line in pediatric hemodialysis: evidence-based practice guidelines. Egyptian Nursing Journal, 14(2): 87-99.

- Naritata, S., Wilcox, M., \& Rafiei, F. (2017). Quality of life in Hemodialysis children. Iranian Patients. Journal of the American Society of Nephrology: JASN, 24 (2), 293-301.

- Peimani, M., \& Pajouhi, M. (2010). Nurse's role in diabetes care; review. Iranian journal of Diabetes and Lipid Disorders, 9, 1-9

- Pitman, N.J. (2013). Pediatric ESRD hemodialysis fact sheet. American Nephrology Nurses Association. Retrieved from: http://www.annanurse.org.
- Talaat, M., Kandeel, A., Rasslan, O., Hajjeh, R., Hallajai, Z., EL-Sayed, N., \& Frank, J. (2006). Evaluation of infection control in Egypt achievements and challenges. American Journal of Infection Control, 34(4): 193-200.

- US Renal Data System (USRDS) (2010). Annual data report: atlas of chronic kidney disease and end-stage renal disease in the United States. Bethesda, MD: National Institutes of Health, National Institute of Diabetes and Digestive and Kidney Diseases. Retrieved at: http://www.usrds.org/adr.htm. [Accessed 13 June 2011].

- Yanai, M., Uehara, Y., \& Takahashi, S. (2006). Surveillance of infection control procedures in dialysis units in japan: A preliminary study. Therapeutic Apheresis and Dialysis Journal, 10(1): 78-86. 